\title{
LAS RELACIONES INTERNACIONALES DE LOS GOBIERNOS LOCALES: EL CASO DE CHIAPAS
}

\author{
Sergio Rodríguez Gelfenstein
}

\section{Introducción}

$\longrightarrow$ omo fuerza imperante en la última década del siglo xx, la globalización ha sido dominada por los aspectos económicos y por la expansión de los mercados. Sin embargo, este proceso puede llegar a tener un rostro humano si se logra una nueva aproximación de los gobiernos, una que preserve las ventajas ofrecidas por los mercados globales y la competencia, pero que permita al mismo tiempo que los recursos humanos, comunitarios y ambientales aseguren que la globalización trabaja para los pueblos y no para las ganancias.

Aquí lo político juega un papel trascendental. Hoy, quien hace política tiene que ocuparse de temas como la corrupción, el narcotráfico, la protección del ambiente, las minorías, la pobreza y la exclusión. Además, la presión del mercado para que el Estado limite su accionar a áreas estrictamente administrativas, ha obligado al hacedor de la política a iniciar un proceso de modernización que le permita mantenerse como actor importante, en un momento en

Sergio Rodríguez Gelfenstein, Oficina de Relaciones Internacionales, gobierno del estado de Chiapas. el que el mercado no ha podido legitimarse como referente valorativo-normativo de las sociedades.

Éste es el marco para que la democracia cobre su verdadero valor, poniendo al ser humano en el centro de su interés y de su acción. La democracia no debe ser entendida como un punto de llegada, sino como un camino que se debe transitar, en el cual, asumir las transformaciones de la política permitirán que ese recorrido sea más provechoso, garantizando el espacio para el libre desarrollo de la actividad de los hombres. Las nuevas alianzas que se realizan bajo el signo de la democracia deben hacerse siempre sobre la base del máximo consenso. En todo caso, el llamado a la participación resulta por demás indispensable para la consolidación de este proyecto democrático, sin embargo, no hay que obviar que las tendencias en contra de la organización de la política de ese modo se revelan muy persistentes en nuestros días, obligándonos a permanecer siempre atentos a las repercusiones que esto pueda tener. Esta situación obliga a que el discurso democrático actual en América Latina conlleve signos que expresen la voluntad de apertura hacia la participación popular, como muestra real de la voluntad política de hacer cambios; esto es, practicar y 
reconocer la política democrática como un vínculo permanente entre los ciudadanos y el gobierno. Éste establece y reconoce las libertades civiles, los derechos políticos básicos, el principio de la mayoría y los derechos de las minorías, elecciones libres y el respeto total a los derechos humanos para la regeneración de la vida ciudadana, el fortalecimiento de las organizaciones intermediarias entre las que destacan los partidos políticos, pero también los sindicatos, las cooperativas, las cámaras patronales y otros grupos de interés organizados en la sociedad.

\section{El inicio de una nueva etapa para México}

Una visión de este cambio expresa que

...aunque la historia de México es larga y en constante transformación, en las últimas décadas se han producido importantes cambios muy acelerados en todos los sentidos. El modelo económico ha cambiado; la estructura autoritaria está cediendo frente a la democracia; el nivel de conciencia social ha avanzado considerablemente; en la cultura, también se dan cambios; México está dejando de ser una sociedad tradicional y de valores humanistas acendrados, para empezar a adoptar los patrones consumistas y pragmáticos de la sociedad moderna globalizada. ${ }^{1}$

Esto permite afirmar que "con la alternancia producida por los sufragios del 2 de julio del año 2000, México ha dejado atrás el singular sistema de partido prácticamente único y ha entrado en el nuevo siglo estrenando un orden político democrático". ${ }^{2}$ El significado de este hecho es trascendente para el país porque abre todas las posibilidades de cambio hacia la democracia plena, respetuosa de las leyes y de la justicia social.
El arribo de un empresario de oposición al poder representó no sólo la continuidad en las políticas de libre mercado, sino también la profundización de las mismas. Para atraer capitales, se cumplirá con todas las exigencias de la economía global. Sin embargo, el Gobierno Federal se ha pronunciado por las reformas del Estado y de ser necesario de la Constitución para establecer en México el estado de derecho, la vigencia de los derechos humanos y la democracia, con lo cual se estaría en mejores condiciones para entrar al concierto de las naciones y beneficiarse con la llegada de capitales al país.

Esta apertura democrática también significó la puesta en marcha de un nuevo federalismo que poco a poco dejará a un lado el régimen presidencialista, que ha caracterizado la forma de gobierno de México, y que está encaminado al equilibrio de las relaciones políticas y administrativas que hasta el sexenio pasado favorecían al poder central en detrimento de los estados y los municipios. El federalismo naciente tiene como fuente principal la fuerza creativa de la sociedad civil, la democracia participativa en donde la propia sociedad, partiendo de las características particulares y de las necesidades de desarrollo de cada entidad, es la generadora de proyectos benéficos para sus estados y por ende para sus habitantes, el ciudadano se debe considerar parte activa del proceso de gobierno y no mero receptor de beneficios o cortesías administrativas; aquí la única tarea de los gobiernos federal y estatal se limita a vigilar que los nuevos equilibrios de poder no marginen o excluyan a grupos, regiones y comunidades. Así funciona un verdadero federalismo.

Como parte de este nuevo pacto federal, el gobierno empieza a descentralizar la administración pública de modo que se va teniendo instituciones más autónomas y con mayores posibilidades de cumplir cabalmente sus funciones en cada estado. Con 
la autonomía política, es decir, la transferencia de atribuciones, recursos, información y decisiones, los gobiernos locales adquieren mayor consistencia y se permite a la vez que éstos lleven a cabo el plan de gobierno sin obstáculos y sobre todo se reafirma el principio de la igualdad jurídica.

Por otro lado, en el ámbito internacional, según expresara la Ministra Rosalba Ojeda:

...se ve a México como un país que se está adecuando a los ritmos que marcan los valores humanos universales a las tendencias de una economía global abierta y en constante competencia y a las innovaciones tecnológicas de los últimos tiempos. Se le percibe también como una nación que dio un paso trascendental en la consolidación de un sistema democrático, donde existe una sana alternancia del poder y un deseo sincero para mejorar las condiciones de vida de todos los ciudadanos. ${ }^{3}$

México es, según se ve ahora, una nación en la que se respetan y garantizan las decisiones políticas de la población, una más que se ha sumado a los esfuerzos democratizadores en América Latina. Como prueba de esta visión internacional, se han intensificado las relaciones con las demás naciones, ratificando el apoyo de éstas al nuevo gobierno y manifestando sus deseos de cooperar con la nación en la conformación de tratados internacionales o como donadores de cooperación en las áreas educativas, culturales, científicas y tecnológicas, en el otorgamiento de financiamiento para los proyectos de desarrollo social y en el refrendo de las relaciones diplomáticas.

\section{Chiapas: Un nuevo gobierno}

En este nuevo contexto, el estado de Chiapas también se abrió a la democracia y el partido que du- rante años había dominado la entidad fue derrotado por la alianza opositora. Observo que el nuevo gobierno ${ }^{4}$ se ha propuesto iniciar un periodo de transición dentro de la creciente democracia, trabajando sobre la base de las características propias del estado y estableciendo políticas sociales de carácter redistributivo que pongan el aparato estatal al servicio de las mayorías, logrando su integración en la federación e insertándose en la dinámica internacional actual.

Percibo, por igual, que las prioridades del estado de Chiapas se orientan hacia la solución de los ingentes problemas de pobreza, educación y salud. Para llevar adelante estos objetivos se necesita atraer inversiones encaminadas a capitalizar el campo, generar agroindustria y detonar otras actividades como la pesca y el turismo. En este marco, se necesita revitalizar la infraestructura vial, de transporte y comunicaciones y generar un nuevo estado de derecho que ponga en el centro el desarrollo del hombre en su relación con el ambiente.

De acuerdo con la anterior, el gobierno de Chiapas ha puesto su atención en las condiciones especiales del estado, como el territorio meridional de México con mayor extensión en términos de frontera: de los 1138 kilómetros que tiene la Frontera Sur, 962 son compartidos entre Chiapas y Guatemala; 16 municipios, de los 21 considerados en la franja, pertenecen a la entidad y ésta es por excelencia el paso natural hacia Centroamérica, no hay que obviar que las relaciones entre ambos territorios no se restringen a la línea fronteriza, pues trascienden los estrechos límites de los municipios que la conforman para ver tierra adentro una serie de fenómenos que son resultado de la interacción con los países centroamericanos. Además, la frontera no se puede considerar sólo como línea para el cruce de mercancías sino también de fuerza de 
trabajo, de "mercancía humana", que se interna en territorio mexicano en busca del sueño blanco americano.

Chiapas posee una ubicación geoestratégica privilegiada por su cercanía con los países vecinos de Centroamérica y su posición frente a la Cuenca del Pacífico, ámbito en el que se desarrollan las principales corrientes del comercio internacional. Con los primeros, comparte características culturales similares que constituyen ventajas comparativas que no se deben soslayar. Con los países y regiones de la Cuenca del Pacífico, la entidad se ha convertido en foco de interés en particular de inversionistas del sudeste asiático que la han visitado para detectar oportunidades de inversión, entre las que destacan la acuicultura y el comercio.

Hasta ahora, la globalización ha representado para Chiapas la profundización de las condiciones de subdesarrollo y de miseria de grandes sectores de la población. Está significando el resquebrajamiento del precario equilibrio de los productores rurales, llegando a trastocar el tejido social como consecuencia de los conflictos provocados por la crisis económica y política.

En un grado importante, el futuro de Chiapas está marcado por las grandes definiciones de la economía mundial, por la vía de la política económica y social del Estado mexicano. En este sentido, de seguir las tendencias actuales, sólo le queda aprovechar los marcos legales, la buena razón y la inteligencia para encontrar una mejor posición en la globalización excluyente. Existen las condiciones, según ilustraré a continuación, para que el estado de Chiapas se inserte con mejores perspectivas en la comunidad internacional.

\section{El marco internacional}

El fin de la guerra fría y el inicio del proceso global han incidido en la formulación de nuevas propuestas para estructurar el sistema internacional en las nuevas condiciones. Ya en 1980, cuando aún imperaba el sistema bipolar, la irrupción de nuevos actores llevó a que el derecho internacional, haciéndose eco de esta nueva tendencia, aceptara en la Convención de Viena sobre el Derecho de Tratados, en su artículo 3 , que el hecho de que esta Convención no se aplique a acuerdos internacionales "celebrados entre Estados y otros sujetos de Derecho Internacional o entre esos otros sujetos de Derecho Internacional..." no afectará ni el valor jurídico de estos acuerdos ni la aplicación de los mismos, tampoco la relación entre estados cuando en ellos formen parte otros sujetos de Derecho Internacional. ${ }^{5}$

El devenir del tiempo dará cuenta de las nuevas particularidades en la conformación de un nuevo sistema internacional. Europa, nuevamente, marcha a la cabeza en el proceso de desarrollo de estas tendencias y en la conformación de un cuerpo político y jurídico que asuma y acepte las mismas. Sin embargo, el procedimiento que lleve a consolidar la comprensión de una nueva legalidad internacional se da en el marco de condiciones muy contradictorias, signadas por la integración, de un lado, y la pérdida de influencia de los Estados nacionales, de otro. Dos fuerzas pugnan en este último aspecto, la de las grandes empresas transnacionales que pretenden "pescar en río revuelto" y la de las identidades regionales y locales organizadas políticamente en regiones, estados, provincias o municipios.

Estas identidades que se sustentan en particularismos culturales se han activado y han ido profundizando en su institucionalización a la par que van cobrando cada vez más presencia en el tablero 
internacional. Esta situación, sin embargo, debe canalizarse por vías institucionales so peligro de transformarse en un evento caótico que conduzca hacia la atomización del orden internacional. Para evitar un resquebrajamiento desordenado que podría llegar a cauces violentos o en su caso más extremo a la guerra como en Yugoslavia o la Unión Soviética, el sistema internacional debe dar cuenta de esta transformación y hacer los reajustes políticos y jurídicos acordes con esta nueva realidad. La pérdida de poder del Estado-Nación se ha asociado a la caída del Muro de Berlín, que ha puesto fin a un sistema internacional en el cual lo ideológico era su elemento ordenador. El Estado nacional todavía traza los contornos de las relaciones que los individuos establecen con sus pares de otros lugares del planeta estableciendo lo que Badie y Smouts llaman "equilibrio funcional" entre los individuos y el orden mundial, ${ }^{6}$ sin embargo, la crisis de identidades pone en duda ese proceso y por tanto las posibilidades de integración que han sido esbozadas.

El debate teórico ha tomado en cuenta esta discusión desde hace bastante tiempo. Durante muchos años, la diversidad de actores que componen el sistema global se enmarcaban en estudios que se hacían desde una perspectiva interestatal. ${ }^{7}$ Otros, como Hans Morghentau ${ }^{8}$ y o Kenneth Waltz, ${ }^{9}$ consideraban las relaciones de fuerza como el único determinante de la política exterior y principio ordenador de la misma. Sin embargo, la noción de actor sigue siendo confundida con la de Estado soberano como único detentador del monopolio de la violencia en el espacio de su territorio. Después de la segunda guerra mundial, fueron los marxistas quienes por primera vez hablaron de fuerzas internacionales exteriores al sistema bipolar. A partir de la década de los cincuenta, behavioristas, y después teóricos de la decisión, neofuncionalistas y teó- ricos de la integración expresaron diferentes opiniones en torno al tema de los actores internacionales, correspondiendo a los teóricos de la decisión el mérito de haber sido los primeros en cuestionar el papel del Estado como único actor del sistema internacional. Sin embargo, no fue sino hasta los años setenta que se reconoció plenamente la existencia de otros actores. ${ }^{10}$ Los trabajos de Robert Keohane y Joseph $\mathrm{Nye}^{11}$ marcaron un punto de inflexión en este proceso al transformar la variable que define a un sistema internacional. Expusieron que ésta era el número de actores a diferencia de aquella que aceptaba que la misma se sustentaba en un número de estados y al referirse a éstos precisaba que eran "aquellos cuyas decisiones afectan recursos y valores y cuya acción de unos sobre otros se ejerce más allá de las fronteras". ${ }^{12}$

Esta nueva definición cambió los puntos de vista respecto a la discusión sobre el tema. Entre otras cosas se podría aceptar que no todos los Estados son actores internacionales, en tanto no tienen acceso a las redes de decisiones políticas, culturales, financieras o de tecnología. Por otro lado, el aumento de 51 Estados que dieron origen a las Naciones Unidas en 1945, a casi 200 que son sus miembros actuales, unido a todas las transformaciones políticas que trajo como consecuencia el fin de la guerra fría y la desaparición de la Unión Soviética -uno de sus pilares fundamentales-, ha estado conduciendo al surgimiento de distintos y novedosos actores. Los mismos van desde individuos, organizaciones delictuales, redes empresariales, de consumidores, hasta líderes religiosos que se venían a sumar a los que ya existían como las Organizaciones No Gubernamentales, organizaciones políticas internacionales, de la Iglesia y las autoridades locales y regionales que en esta nueva realidad comienzan a cobrar fuerza y presencia en el escenario internacional. Su principio 
de funcionamiento es diverso, desde su actuación bajo el imperio de la ley o al margen de ésta y su criterio valorativo de la racionalidad política, jurídica y ética es también variado.

Esta certidumbre ha venido a producir una verdadera revolución en las relaciones internacionales, la cual, lamentablemente, algunos no toman en cuenta. Desde el punto de vista teórico se han hecho importantes avances. Se ha comenzado a reconocer lo que se ha dado en llamar susbsistemas internacionales. Uno de ellos, el que interesa para efectos de este trabajo, es el subsistema regional que agrupa a todas aquellas autoridades locales, regionales y de ciudades que recurren al sistema internacional para suplir las falencias en sus procesos de construcción institucional y de desarrollo económico. Aquí, puedo utilizar, como elementos para distinguirlo, una extensión espacial determinada, la existencia del subsistema como entidad distinta, una conciencia de tal situación por parte de sus miembros y la existencia de una red de interacciones relativamente autónoma de sus integrantes. ${ }^{13}$

Para buscar una aceptación en las contradicciones que para el sistema internacional actual ha generado esta propuesta teórica, James Rosenau ${ }^{14}$ propone un cambio de óptica para el estudio de los actores internacionales. Rosenau conceptualiza la política mundial en tres dimensiones: un nivel micro, para los individuos, un nivel macro para la acción colectiva y un nivel mixto, en el cual se articulan las relaciones de autoridad de los precedentes. El gran aporte de Rosenau es exponer que hay un fenómeno de bifurcación en el cual coexisten los dos modelos de política mundial: el Estado-céntrico que es el actual, surgido desde 1648 a partir del Tratado de Westfalia, pero que ha ido perdiendo fuerza, y el multicéntrico, más flexible y más libre en sus modalidades de acción, el cual da cuenta de los cambios en el sistema internacional, lo amplía y lo hace más democrático. A partir de esto, Rosenau expone que el sistema internacional debe construirse en torno a dos variables: los actores y los ámbitos que se abarcan. Así, el sistema global será el de las organizaciones que tengan que ver con un escenario y una agenda más amplia y los subsistemas serán aquellos que manejen acciones restringidas a una gama de ámbitos. Este espacio debe ser aquel en el cual concuerden los intereses y la problemática que debe resolverse. Rosenau concluye que las relaciones internacionales no deben seguir analizándose a partir de la soberanía y los recursos, es decir del Estado y su potencia, sino a través de la identificación de actores y la apreciación de resultados, tratando de identificar las estructuras de autoridad, su espacio de acción, aclarando las consecuencias que se derivan del ejercicio de la autoridad. ${ }^{15} 16$

\section{La experiencia de las relaciones internacionales de los gobiernos locales y regionales}

Esta situación de la que los teóricos han tomado nota y que el derecho internacional ha comenzado a asumir, ha avanzado mucho más aceleradamente desde el punto de vista estructural y organizativo. Estas autoridades de las que habla Rosenau han patentizado desde hace algunos años la búsqueda de soluciones a sus problemas comunes, por otra parte, han hecho esfuerzos por agruparse a partir de sus especificidades geográficas, temáticas o de una misma lengua. Así trabajan en común temas como el transporte, la salud, la lucha por la paz, las grandes ciudades, las ciudades mártires y otros innumerables aspectos. Esto las ha llevado a encarar una lucha por lograr su reconocimiento ante sus autoridades nacionales y ante los organismos internacio- 
nales. En este ámbito se ha planteado la necesidad que estas instancias internacionales las tomen en cuenta para el diseño de sus políticas, sobre todo aquellas que las afectan directamente. Bajo el manto de la colaboración internacional las relaciones internacionales de las autoridades locales se llevan a cabo a través de dos vertientes, por una parte se debe mencionar el gran esfuerzo por abrirse un espacio de representación institucional en el sistema de Naciones Unidas y por otro se ha desarrollado un trabajo más dinámico sustentado en la idea de fortalecer la autonomía de dicho movimiento, es decir, hacer lo mismo sin integrarse a los esquemas de las organizaciones internacionales preexistentes. ${ }^{17}$

Es menester indicar que para llevar adelante la primera vertiente se ha hecho un trabajo encaminado a que el Centro de Naciones Unidas sobre Asentamientos Humanos (CNUAH/Hábitat), con sede en Nairobi, Kenya, acepte estas asociaciones en su seno. Así, en el marco de la Conferencia de Naciones Unidas sobre Asentamientos Humanos, celebrada en Estambul, en junio de 1996 (Hábitat II), se reunió la Primera Asamblea Mundial de Ciudades y Gobiernos Locales con la presencia de más de 500 autoridades de gobiernos locales de todos los continentes; se declaró el compromiso de crear un foro único de representación internacional de los gobiernos locales del mundo, dando paso de esa forma a la creación de la Coordinación Mundial de Asociaciones de Ciudades y Gobiernos Locales (Camcal). Ésta ha asumido la responsabilidad de transformarse en la organización que represente a las organizaciones locales ante Naciones Unidas. Sin, embargo al tener status de observador carece de derecho a voto en las decisiones que toma el Centro de Naciones Unidas sobre Asentamientos Humanos. ${ }^{18} \mathrm{La}$ Camcal se propone promover la autonomía local, preparando para ello una Carta Mundial de Gobiernos Locales para ser promulgada por la ONU; fortalecer la participación de las ciudades y autoridades locales en el ámbito internacional y mejorar la cooperación internacional hacia las ciudades y autoridades locales. ${ }^{19}$

Respecto a la segunda forma de proceder, es decir, a través del intento por desarrollar un modelo autónomo en el espacio internacional, el contexto actual señala un proceso hacia la unificación de las dos grandes organizaciones internacionales de gobiernos locales, la Federación Mundial de Ciudades Unidas (FMCU) y la Unión Internacional de Autoridades Locales (IULA). Ambas existen desde hace varias décadas y cuentan con una importante membresía a través de todo el planeta. La nueva organización mundial de gobiernos locales unifica$\mathrm{da}^{20}$ se propone ser la vOz de los gobiernos locales en el ámbito mundial; consolidar una representación política eficaz de los gobiernos locales ante la Comunidad Internacional; asegurar una carta mundial sobre la Democracia Local que sea adoptada por los Estados miembros de la Organización de las Naciones Unidas (ONU); ser fuente de información para los gobiernos locales; ser fuente de educación, capacitación e intercambio entre los gobiernos locales y sus asociaciones nacionales; promover el desarrollo social, económico, ambiental y cultural de la población; fomentar la equidad de raza y género, combatiendo todas las formas de discriminación; conformar una organización fuerte y democrática que refleje la diversidad de todos sus miembros; promover la colaboración y los hermanamientos como medio de reconocimiento recíproco y amistad entre sus miembros; definir políticas y desarrollar programas e iniciativas buscando los recursos apropiados para ello y promover la cooperación descentralizada y la cooperación entre gobiernos locales. ${ }^{21} 22$ 
Vale mencionar que los hermanamientos y la cooperación entre ciudades es una práctica que se ha dado con regularidad desde la década de los cincuenta, especialmente entre centros urbanos y poderes locales. En relación con la "cooperación descentralizada", la cual se va transformando en una de las principales y más novedosas formas de cooperación, dicha práctica sitúa a los actores locales en el centro del proceso de cooperación, estableciendo los roles y responsabilidades de cada actor. ${ }^{23} \mathrm{Sin}$ embargo, es importante mencionar que en este concepto cohabitan dos concepciones: la primera habla de la cooperación internacional entre entes públicos territoriales. En la segunda, se maneja la idea de que la cooperación descentralizada es la forma más amplia, horizontal y participativa, es una fórmula que permite la interacción de una extensa gama de actores locales como ONG, sindicatos, universidades, cámaras de comercio e industria y otros. ${ }^{24}$

\section{Las relaciones internacionales del gobierno de Chiapas}

Este marco conceptual, esta práctica internacional y el contexto político nacional y regional se juntaron en el tiempo y en el espacio y dieron pie para que el gobierno de Chiapas se propusiera establecer pautas de trabajo hacia el escenario internacional. Desde sus inicios en diciembre del 2000, el gobierno del Estado aplica una política internacional de puertas abiertas, que tiene sus primeros frutos en el inicio de relaciones directas con diversos países del mundo, en contraste con el aislamiento de administraciones pasadas en esta materia.

Esta nueva política internacional se respalda en el nuevo pacto con la federación, la que fungirá como el medio que facilite a los estados las oportunidades que se presenten de acuerdo con los tratados inter- nacionales de los que México es parte. Es así que, con fundamento en la Convención de Viena de 1980, se dio un paso trascendental para que los gobiernos estatales puedan celebrar acuerdos interinstitucionales apoyados en el decreto del 2 de enero de 1992, relativo a la ley sobre la celebración de tratados, el cual faculta a las dependencias u organismos descentralizados de la administración pública federal, estatal o municipal para establecer convenios con uno o varios órganos gubernamentales extranjeros u organizaciones internacionales, cualquiera que sea su denominación, sea que derive o no de un tratado previamente aprobado. En cualquier caso, la Secretaría de Relaciones Exteriores del Gobierno Federal debe ser informada al respecto. ${ }^{25}$ Aunque no lo dice expresamente, al decretar esta ley, el gobierno federal le estaba concediendo a las dependencias $u$ organismos descentralizados de la administración federal, estatal o municipal, la posibilidad de transformarse en sujetos de Derecho Internacional. En la práctica aún no lo son, porque todavía se mantienen modalidades de control federal en torno a la firma de un acuerdo interinstitucional, pero esta ley, que significa un gran paso adelante en el proceso de descentralización federal, también le da a México una posición de vanguardia en la evolución hacia un sistema internacional más participativo y democrático.

El gobierno del presidente Vicente Fox, a través de la Secretaría de Relaciones Exteriores (SRE), creó la Dirección General de Enlace Federal y Estatal (DGEFE). Ésta es la unidad administrativa de la SRE encargada de facilitar y mejorar la coordinación de la cancillería con los estados y municipios, apoyando y cooperando con los gobiernos estatales y municipales en sus actividades internacionales. Aprovechando la propia experiencia desarrollada, pero también la de otros países como Brasil, Canadá y Francia, esta institución se sustenta en la constatación de que los 
estados y municipios han adquirido una creciente presencia internacional, la cual requiere -desde la competencia federal- ser estimulada y encauzada de forma tal que las acciones que se realicen por estas instancias se ajusten al marco normativo vigente en la materia. Aunque la DGEFE es de nueva creación, ya se han planteado importantes objetivos encaminados a proveer información a los gobiernos estatales y municipales para la realización de sus actividades internacionales, servir de enlace institucional entre los gobiernos de los estados y municipios con la Secretaría de Relaciones Internacionales (SRE), dar seguimiento a las peticiones que los gobiernos regionales y locales hagan a la Secretaría de Relaciones Internacionales (SRE) en función de desarrollar sus actividades internacionales, proporcionar asesoría en materia de cooperación internacional y para la participación de los gobiernos regionales y locales en eventos internacionales, apoyar a los gobiernos locales y regionales para la configuración de las agendas en sus viajes al exterior, así como atender las solicitudes de funcionarios e instituciones extranjeras que deseen visitar estados y municipios de México.

En este nuevo marco político nacional, observo que el reto para Chiapas es lograr su inserción y posicionamiento internacional, reduciendo al máximo los costos en niveles de vida de la población, en desempleo, en destrucción de recursos naturales y en democracia. Partiendo de las riquezas internas que se tienen tanto en su gente, como en los recursos naturales, el gobierno chiapaneco plantea convertir la cooperación internacional y el financiamiento externo en recursos complementarios a los esfuerzos de desarrollo internos.

Hay que tomar en cuenta que, a partir de 1994, Chiapas se convirtió en un imán para el mundo. Grupos de activistas, ambientalistas, organizaciones internacionales de derechos humanos y de ayuda humanitaria, empresarios, iglesias y una gran cantidad de intelectuales de universidades de varios países han seguido de cerca el proceso chiapaneco. Chiapas es quizás el territorio más estudiado y visitado en los últimos años por personalidades de gran prestigio: Premios Nobel, representantes de las $\mathrm{Na}$ ciones Unidas, embajadores de los países del continente, de la Unión Europea y de otras regiones, así como escritores y artistas de gran renombre.

Chiapas no es un estado industrializado o con un alto grado de desarrollo agroindustrial que pueda ofrecer al mundo una gama de bienes bajo criterios de eficiencia y competitividad. Por el contrario, sus relaciones con los mercados internacionales están centradas en pocos productos: el café, el banano y algunas frutas tropicales. Sin embargo, puede ofrecer una gama muy amplia de aspectos que tienen que ver con las riquezas naturales y culturales: diversidad biológica, sitios naturales, regiones con amplia diversidad de condiciones climáticas y culturales, e importantes sitios arqueológicos.

\section{Notas}

${ }^{1}$ Vigil Avalos, Gabriel, www.arquidiocesisgdl.org.mx ${ }^{2}$ Salazar, Luis; "Frívolos y Cursis", en Nexos, abril 2001, Núm. 280, p.11.

${ }^{3}$ Palabras en la Universidad Autónoma de Chiapas, Tuxtla Gutiérrez, Chiapas; 12 de junio del 2001.

${ }^{4}$ El 8 de diciembre de 2000 asumió un nuevo gobierno la conducción del estado de Chiapas, después de haber ganado las elecciones del 20 de agosto. El nuevo gobernador, Pablo Salazar Mendiguchía, construyó en torno a la agrupación de ciudadanos "Movimiento de la Esperanza" una amplia alianza configurada por ocho partidos que logró desalojar al Partido Revolucionario Institucional del poder que había conservado por 71 años.

${ }^{5}$ Rodríguez, Sergio: "Relaciones Internacionales, Estado Nacional e identidades en el siglo que comienza" en Rodríguez 
Sergio (coord.) La posibilidad de seguir soñando, Gijón, Asturias, Literastur, 2000.

${ }^{6}$ Badie, Bertrand y Marie-Claude Smouts, Los operadores del cambio en la política mundial. Sociología del escenario internacional, México, Publicaciones Cruz O.S.A./Fundación Nacional de Ciencias Políticas de Francia \& Dalloz, Primera edición en español, 2000.

7 Aron, Raymond 'Qu'est-ce qu'une théorie des relations internationales?, Revue francaise de science politique, 17 de octubre de 1967.

${ }^{8}$ Ver Morgenthau, Hans.J., "La política entre naciones", Teoría Contemporánea de las relaciones internacionales, Serie Recopilaciones Núm. 2 Escuela de Estudios Internacionales, Caracas, 1986. ${ }^{9}$ Ver Waltz, Kenneth N. Theory of International Relations, Mc Graw Hill, New York 1976 y The Emerging Structure of International Politics, en: Brown et al. (eds.) The peril of anarchy, The мIT Press 1995.

${ }^{10}$ Merle, Marcel, La crise de L'Etat- nation en Forces et en enjeux dans les relations internationales, París, Económica 1981.

${ }^{11}$ Ver Transnational Relations and World Politics, Cambridge, Mass, Harvard University Press,1972.

${ }^{12}$ Definición propuesta por Stanley Hoffman en referencia a Keohane y Nye en Le dilemme américain: suprématie ou ordre mondial. París Económica 1982.

${ }^{13}$ Esta excelente definición está tomada de Badie y Smouts (op.cit.) y combina los principales criterios propuestos por M. Brecher y E. Haas. Para profundizar ver Singer, J.D. y M. Small "Systeme et crise en politique international" en Korany Baghat et. al., Analyse des relations internationales approches, concepts et donées, Gaeten Morin, Montreal, 1987.

${ }^{14}$ Rosenau, James Turbulence in World Politics: A theory of change and continuity, Princeton University Press, Princeton 1990 y Rosenau, James y Mary Durfee Thinking theory throughly. Coherent aproaches to an incoherent world. Westview Press, Boulder 1995.

${ }^{15}$ Rosenau, James, The Scientific Study of Foreign Policy, Londres, Frances pinter, 1980 o Linkage Politics: Essays ONU the convergence of national and international systems, Nueva York, Free Press, 1969.
${ }^{16}$ Para seguir toda esta discusión teórica ver Badie, Bertrand y Marie Smouts (op.cit) y Rodríguez, Sergio "De la modernidad a la postinternacionalización. Un camino para construir una teoría de las relaciones internacionales" en Petrásh, Vilma y Eira Ramos (coords.) Cambio, contradicción y complejidad en la política internacional del fin de siglo. Caracas, FacesuCv/Ceap-Faces-ucv/Conicit 1998).

${ }^{17}$ Zapata- Garesché, Eugene "Los desafíos al asociacionismo municipal” en Zapata-Garesché, Eugene, Carlos Rodríguez Wallenius y Enrique Ortiz Herrasti. Asociaciones de Gobiernos Locales (Cesem/Fmcu México, 2001).

${ }^{18}$ Zapata- Garesché, Eugene, op. cit.

${ }^{19}$ Zapata-Garesché, Eugene, Carlos Rodríguez Wallenius y Enrique Ortiz Herrasti, op.cit. Cap.III.

${ }^{20}$ Los documentos que dan pie a esta decisión fueron aprobados por el Consejo Internacional de la FMCU el 22 de enero de 1999 en Sevilla, España, y por el Wexcom de IULA el 20 de marzo de 1999 en Barcelona, España. Los mismos. ${ }^{21}$ Ibid.

${ }^{22} \mathrm{El}$ documento que aprobó estas ideas fue elaborado por el Comité Negociador Conjunto IULA-FMCU, el cual adoptó la resolución que contiene las bases políticas de la nueva organización. En la misma el término "local" se refiere a municipios, así como a los diferentes niveles o esferas de las entidades públicas infraestatales de acuerdo con las diferentes estructuras político-administrativas nacionales.

${ }^{23}$ Para ampliar estas ideas, ver "City -to- city Cooperation: Issues arising from experience.", documento preparado por Habitat, IULA-FMCU y Camcal para servir como información para las discusiones sobre cooperación descentralizada en el Congreso de unificación de IULA-FMCU que se celebró del 3 al 6 de mayo de 2001 en Río de Janeiro y para la sesión especial de la xxv Asamblea General de Naciones Unidas (Estambul $+5)$ referida a la Cooperación Ciudad a Ciudad en New York del 6 al 8 de junio de 2001 .

${ }^{24}$ Bilsky, Edgardo y Leonardo Díaz Abraham Programa de cooperación descentralizada Europa-América Central (París, Ciudades Unidas Desarrollo, 2000).

${ }^{25}$ Diario Oficial de la Federación, Tomo cDlx, Núm. 1, México, D.F., Jueves, 2 de enero de 1992. 